\title{
Development of microfluidic LED sensor platform
}

\author{
Tae Hyeon Kim ${ }^{1}$, Jong Min Lee ${ }^{1}$, Bong Hyun Chung ${ }^{2^{*}}$ and Bong Geun Chung ${ }^{1 *}$
}

\begin{abstract}
We developed the microfluidic light emitting diode (LED) sensor for methanol detection. The linear gradient-generating microfluidic device consists of two inlet and four outlet microchannels. The concentration gradients of methanol were stably generated in the microfluidic platform in a temporal and spatial manner. The methanol harvested from microfluidic platforms was analyzed by measuring electrical conductivity, showing that currents were decreased with the methanol content. The methanol in the microfluidic device was also observed by LED sensor. Therefore, this microfluidic LED device could be a powerful platform for methanol sensor applications.
\end{abstract}

Keywords: LED sensor; Microfluidic gradient device; Methanol detection; Electrical conductivity

\section{Background}

Methanol, the simplest alcohol and organic solvent, has widely been used for fuel cell and biochemical applications [1-3]. The direct methanol fuel cell is of great benefit for portable electronic devices, because it enables increase of power density and decrease of operating temperature [4-6]. The physical and chemical properties of methanol are also similar to gasoline, showing that methanol is a good candidate as a fuel cell in automotive engines [7-9]. Despite many methanol-based industrial applications, the major problem is still remained, such as methanol toxicity, which can cause severe disease of metabolic acidosis, nerve disorder, olfactory mucosa, and blindness [10]. Although small amount of methanol is inhaled, methanol becomes toxic formic acid via formaldehyde as previously described [11]. In general, the volatile organic compound (e.g., methanol), which can be conventionally detected by gas chromatography technique, has low boiling point and high reactive property. Thus, the development of the platform technology to detect the methanol at room temperature has become imperative [12].

Microfluidic devices have previously been developed to generate concentration gradients and detect biomolecules [13-15]. Recently, an integrated microfluidic device has been developed to quantify methanol concentrations [16]. The methanol and methanol oxidase infused into the poly

\footnotetext{
* Correspondence: chungbh@kribb.re.kr; bchung@sogang.ac.kr

${ }^{2}$ BioNano Heath Guard Research Center, Korea Research Institute of

Bioscience and Biotechnology, Daejeon, Korea

${ }^{1}$ Department of Mechanical Engineering, Sogang University, Seoul, Korea
}

(methyl methacrylate) (PMMA)-based microfluidic device. The injected solutions were heated at $45^{\circ} \mathrm{C}$ to generate formaldehyde and methanol concentrations were subsequently observed by ultraviolet (UV) spectrophotometer. The accuracy of microfluidic device-based methanol detection was approximately $93 \%$ compared to gas chromatography method. Integrated microfluidic device has been developed to detect methanol concentrations [17]. The system consisted of light emitting diode (LED) photometer, PMMA-based microfluidic device, photodiode, voltmeter, and temperature controller. The samples were loaded and were subsequently mixed by a vortex stirrer in the microfluidic device. The colorimetric reaction of the mixtures was performed by thermoelectric cooler and micro-hotplate systems. LED photometer system detected methanol concentrations in the microfluidic device. Furthermore, microfluidic device has been developed for direct methanol fuel cell applications [18]. For the fuel cell, methanol and oxygen was employed as an anode and cathode, respectively. The microfluidic device containing anode flow fields enabled the generation of liquid-gas meniscus. It showed that the interfacial mass-transfer resistance was largely generated by evaporation of the small meniscus, resulting in inhibition of methanol delivery to anode catalyst layers. The flow field enabled the formation of passive direct methanol fuel cells containing higher methanol concentrations. However, previous approaches have used complex integrated microfluidic devices to detect methanol gas at high temperature. To overcome these limitations, we developed the linear gradient-generating microfluidic device to monitor and quantify the methanol 


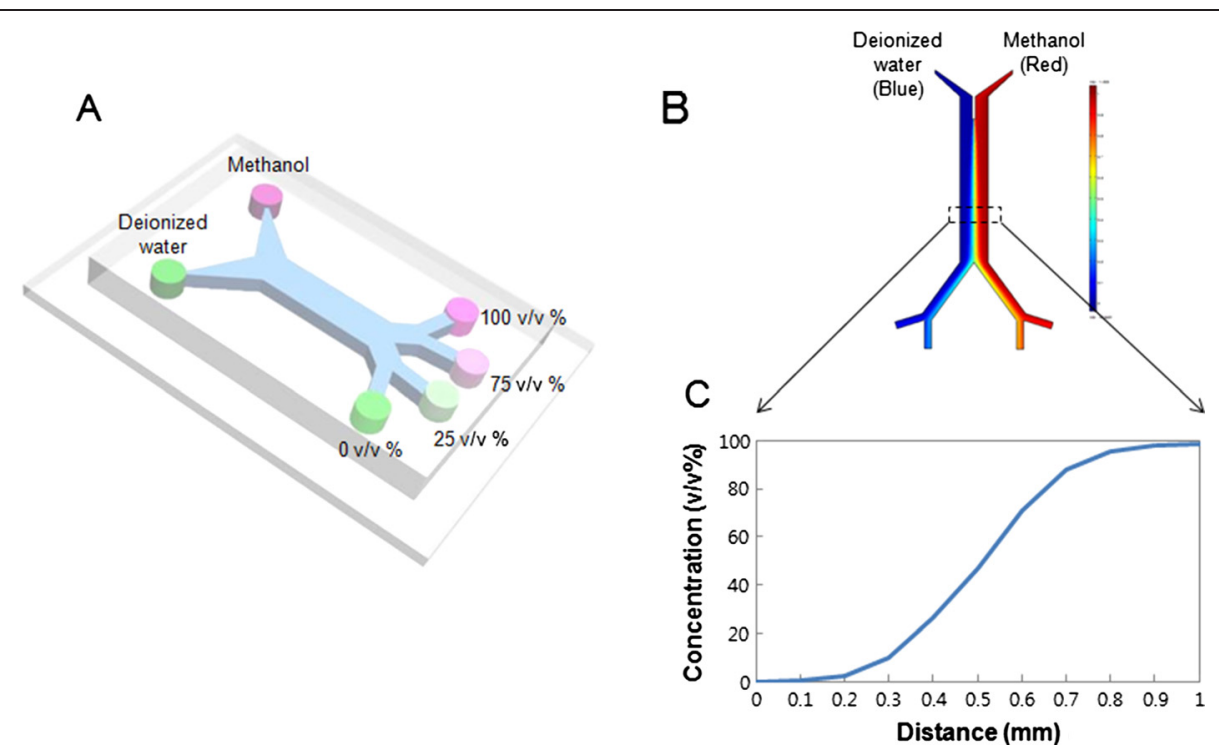

Figure 1 Microfluidic LED sensor platform. (A) Schematic of microfluidic device. (B) Computational simulation of methanol concentration gradients in the microfluidic device. (C) Linear gradient profile of methanol in the microfluidic device. The gradient graph is obtained from the dotted box in Figure 1B.

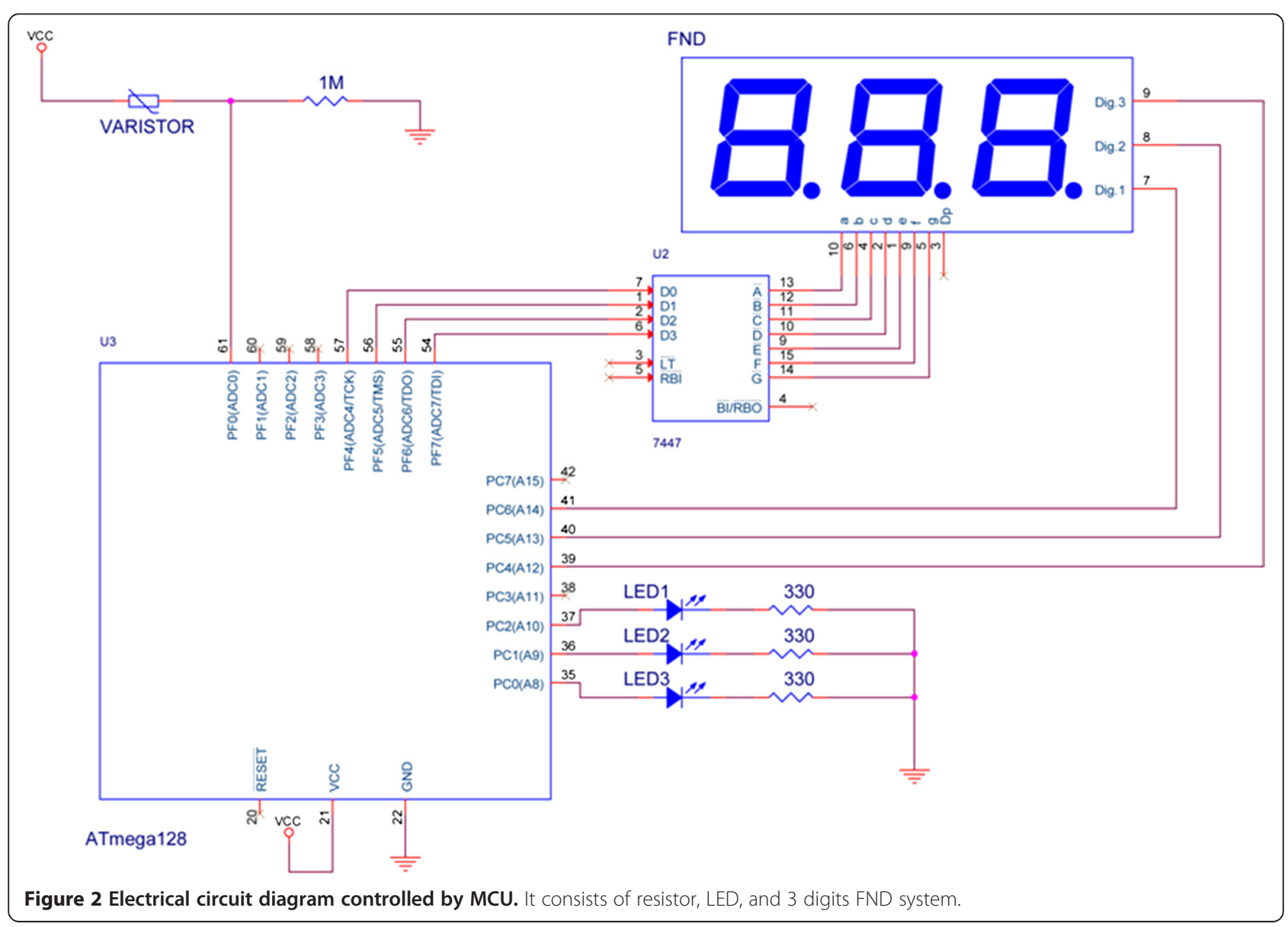


at room temperature using programmed microcontroller, LED sensor, and electrical conductivity analysis. Therefore, this microfluidic LED device could be a powerful tool for methanol sensor applications.

\section{Methods}

2.1 Fabrication of the microfluidic device to generate methanol concentration gradients

We developed the microfluidic device to generate methanol concentration gradients (Figure 1A). Briefly, SU-8 50 (Microchem, USA) negative photoresist was spin-coated onto a silicon wafer to make microchannels with $100 \mu \mathrm{m}$ in thickness. The wafer was pre-baked on a hot plate and the photoresist layer was exposed to ultraviolet (UV) light for 60 seconds through the photomask thin film. The photoresist-patterned silicon wafer was subsequently micromolded using a poly(dimethylsiloxane) (PDMS) elastomer and curing reagent (10:1 mixture). The microfluidic device was bonded to a glass slide after oxygen plasma treatment (Femto Science, Korea).

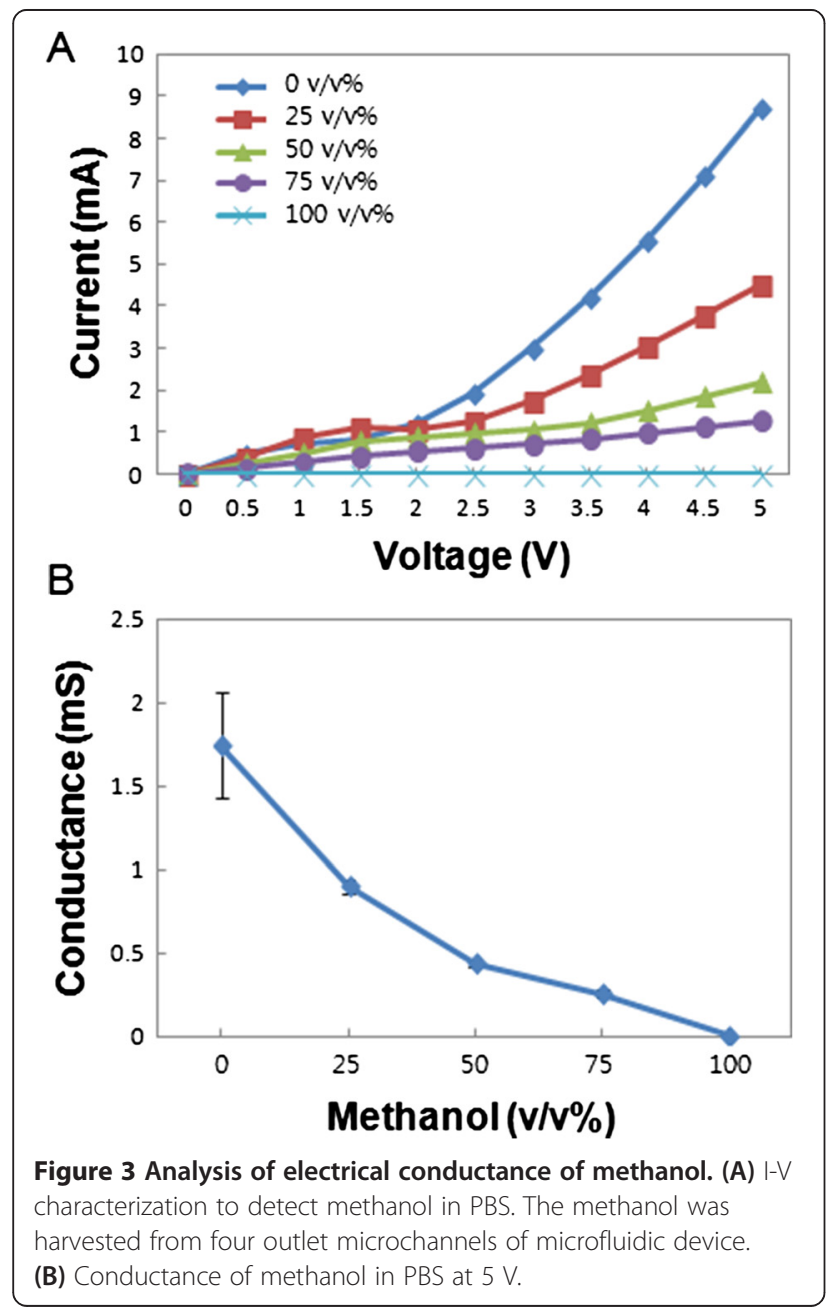

\subsection{Experimental set-up}

The microfluidic device containing two inlets and four outlets allowed streams of solutions to generate laminar flow in parallel without any convective mixing. To generate concentration gradients in the microfluidic device, the methanol and buffer solutions were injected with a uniform flow rate $(66 \mu \mathrm{l} / \mathrm{min})$ using a syringe pump (Harvard Apparatus, USA). The methanol was analyzed by measuring current-voltage (I-V) curve using source meter and Labview programming.

\subsection{Computational simulation}

We conducted the mathematical analysis to optimize the optimal flow rate. The velocity profiles of the fluid as well as convection and diffusion between the flows injected from two inlets were simulated by COMSOL Multiphysics 3.4 software (Figure $1 \mathrm{~B}, \mathrm{C}$ ). To simulate the behavior of the fluids within the microchannel, two partial differential equations were used as follows:

(i) Navier-Stokes equation

(ii)Convection-diffusion equation
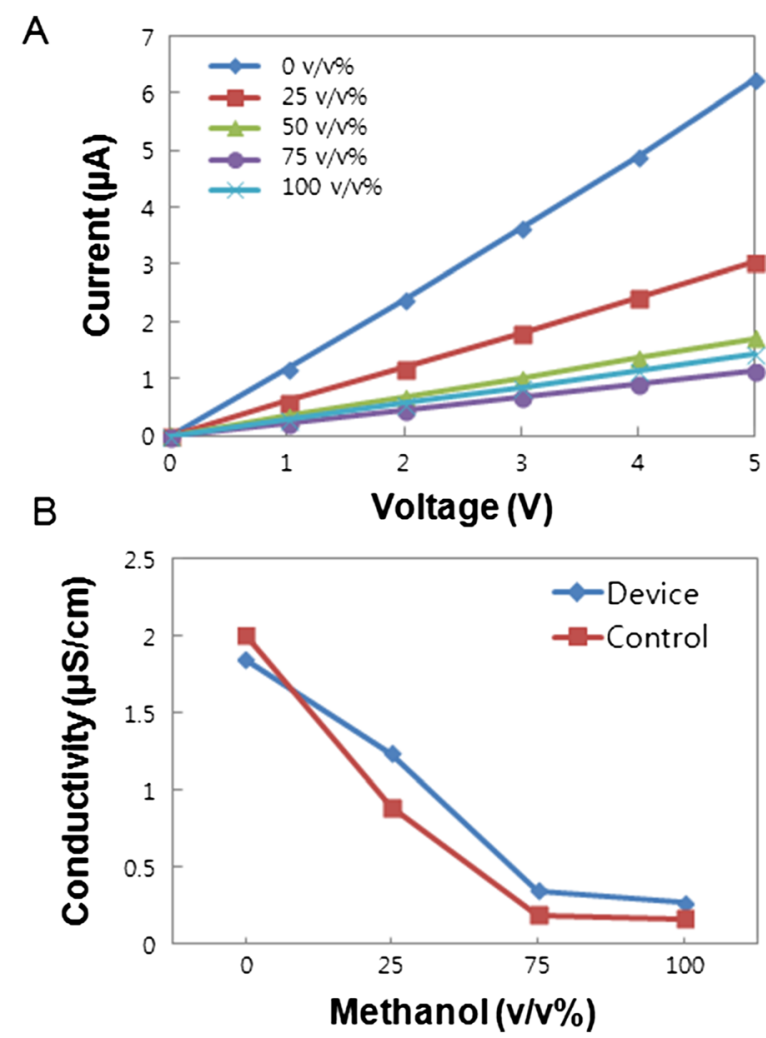

Figure 4 Analysis of conductivity of methanol. (A) I-V characterization to detect methanol in deionized water. The methanol was harvested from four outlet microchannels of microfluidic device. (B) Conductivity of methanol with deionized water at $5 \mathrm{~V}$. 
A

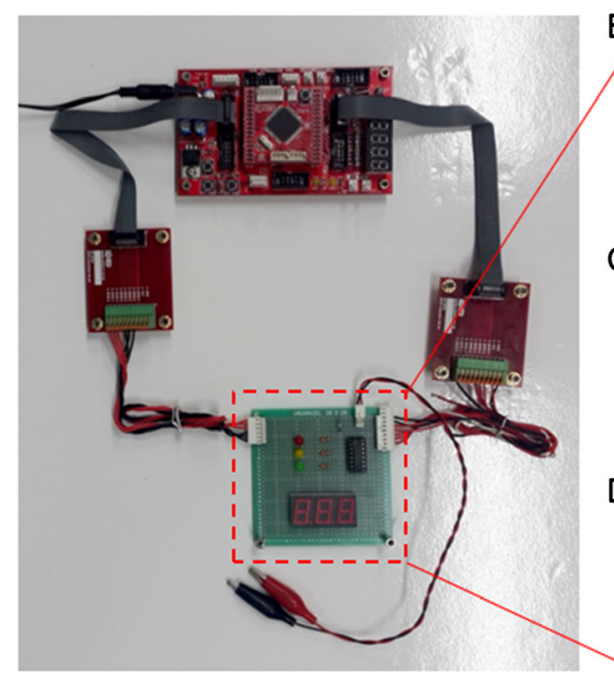

B

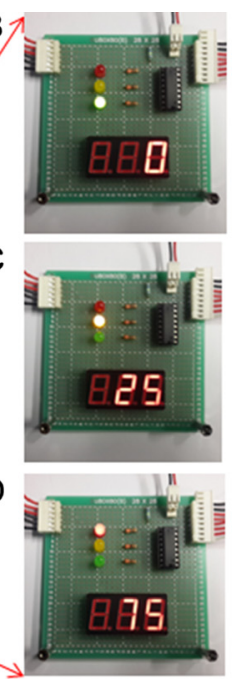

Green LED

(0 v/v\% methanol)

Yellow LED

$(25 \mathrm{v} / \mathrm{v} \%$ methanol)

Red LED

(75 v/v\% methanol)

Figure 5 Sensor operation system containing three LED colors (green, yellow, and red) and 3 digits FND to determine methanol harvested from microfluidic device. (A) The photograph of LED circuit system. (B-D) Photograph of operating LED sensor at 0 , 25, and $75 \mathrm{v} / \mathrm{v} \%$ methanol.

The velocity profiles of fluids in the microchannel were described by solving Navier-Stokes equations derived from the conservation law of mass and momentum in fluid dynamics. These equations for an incompressible Newtonian fluid with constant fluid density $(\rho)$ and kinetic viscosity $(v)$ are given by

$$
\begin{aligned}
& \frac{\partial \mathrm{u}}{\partial \mathrm{t}}+(\mathbf{u} \cdot \nabla) \mathbf{u}=-\frac{1}{\rho} \nabla \mathrm{p}+v \nabla^{2} \mathbf{u} \\
& \nabla \cdot \mathbf{u}=0
\end{aligned}
$$

where $\mathbf{u}$ represents a vector of the flow velocity of the fluid particles in the $\mathrm{x}, \mathrm{y}$, and $\mathrm{z}$ direction and $\mathrm{p}$ is pressure acting on the particles. At two inlets, the velocity generated from syringe pumps in the in-plane direction was set to a constant value $(4 \mu \mathrm{m} / \mathrm{sec})$. Z-directional velocity should be zero due to small thickness of the microchannel compared to its in-plane dimensions. The viscous and normal stress of the outlet was zero and noslip conditions were applied at all the walls of the microchannel. The convection-diffusion equation used for concentration analysis described the convective and diffusive transport of the molecules in the microchannel.

$$
\frac{\partial \mathrm{C}}{\partial \mathrm{t}}+(\mathbf{u} \cdot \nabla) \mathrm{C}=\mathrm{D} \nabla^{2} \mathrm{C}
$$

$\mathrm{C}$ is the concentration, $\mathbf{u}$ is the velocity vector calculated by the Navier-Stokes equations (1) and (2). D is the diffusion coefficient $\left(2.19 \times 10^{-9}\right)$ at the condition of $0.1 \mathrm{MPa}$ and $298.5 \mathrm{~K}$ [19]. The number of elements of mesh used in this simulation was approximately 116,632. Consequently, the optimal flow rate $(66 \mu \mathrm{l} / \mathrm{min})$ generated by a syringe pump was calculated.

\subsection{Electrical circuit diagram for LED sensor}

We designed the electrical circuit diagram to investigate the methanol harvested from four outlet microchannels of microfluidic device (Figure 2). The methanol sensor consisted of LEDs with three colors (green, yellow, and red) and 3 digits flexible numeric display (FND) to determine the methanol. The methanol LED sensor was controlled by 8 bit microcontroller unit (MCU, ATmega128, USA). The microcontroller, which held analog to digital converter (ADC), was operated by computer programming using advanced virtual RISC (AVR) based on C language (Additional file 1). 74HC47 integrated circuits we used for

Table 1 Analog/digital (A/D) conversion voltage used in a microcontroller

\begin{tabular}{lllll}
\hline $\begin{array}{l}\text { Methanol } \\
(\mathbf{v} / \mathbf{v} \%)\end{array}$ & $\begin{array}{l}\text { Conductance } \\
(\boldsymbol{\mu S})\end{array}$ & $\begin{array}{l}\text { Resistance } \\
(\mathbf{M} \Omega)\end{array}$ & $\begin{array}{l}\text { Theoretical } \\
\text { A/D voltage }(\mathbf{V})\end{array}$ & $\begin{array}{l}\text { Experimental } \\
\text { A/D voltage }(\mathbf{V})\end{array}$ \\
\hline 0 & 1.24 & 0.81 & 2.8 & 3.3 \\
25 & 0.61 & 1.64 & 1.9 & 2.6 \\
75 & 0.23 & 3.45 & 1.1 & 1.5 \\
100 & 0.29 & 3.45 & 1.1 & 1.3 \\
\hline
\end{tabular}


LED sensor could convert binary to decimal number to display through FND.

\section{Results and discussion}

We developed the linear gradient-generating microfluidic device to investigate the effect of the methanol on electrical conductivity (Figure 1A). Computational simulations have widely used to analyze concentration gradients in microfluidic device [20,21], temperature gradient of microwave [22], and current distribution in conductor [23,24]. We used the computational simulation to optimize the fluidic velocity $(66 \mu \mathrm{l} / \mathrm{min})$ that could generate linear concentration gradients of methanol inside the microfluidic device (Figure 1B,C). We could not use the fluorescent dye which should be similar molecular weight to methanol, because molecule weight $(32.04186 \mathrm{~g} / \mathrm{mol})$ of methanol was lower. It has been known that the conductance was affected by methanol concentrations in a gas phase [25]. However, it is still not clear the relationship between conductance and methanol solutions at room temperature. We investigated the conductance change of methanol solutions harvested from the microfluidic device. First, we analyzed the conductance of methanol using phosphate buffered saline (PBS) solution (Figure 3). We observed that the current was proportional to voltage (Figure 3A). The conductance was decreased with increasing methanol solutions (Figure 3B). It was probably due to ions inside PBS. We also observed the conductivity of methanol with deionized water using a conductivity meter (Figure 4). Similar to previous PBSbased results, the conductivity was inversely proportional to methanol content (Figure 4B). However, we interestingly observed that the conductivity of $75 \mathrm{v} / \mathrm{v} \%$ methanol was not significantly different to that of $100 \mathrm{v} / \mathrm{v} \%$ methanol due to chemical binding between methanol and water molecules. Water and methanol was generally bound by hydrogen bonding (2:5 ratio) as previously described [26]. When methanol percentage was increased, the molecular binding that could inhibit ionization of molecules was occurred. As a result, the conductivity was decreased until $75 \mathrm{v} / \mathrm{v} \%$ methanol. However, solutions were fully saturated around $75 \mathrm{v} / \mathrm{v} \%$ methanol, because two water molecules and five methanol molecules were bound. There were no water molecules that could bind methanol molecules above $75 \mathrm{v} / \mathrm{v} \%$ methanol. Thus, methanol could be ionized, because methanol molecules could not bind with water molecules. However, the effect of methanol ionization on conductivity might be too subtle to recognize. As a result, the conductivity of $100 \mathrm{v} / \mathrm{v} \%$ methanol was approximately similar to that of $75 \mathrm{v} / \mathrm{v} \%$ methanol. We also developed the MCU-based LED sensor to detect the methanol harvested from four outlet microchannels of the microfluidic device (Figure 5A). 0, 25, and $75 \mathrm{v} / \mathrm{v} \%$ methanol was visualized by green, yellow, and red LED color, respectively. Their numbers of methanol percentage were also shown by FND system of LED sensor
(Figure 5B-D). We observed that LED signal of $100 \mathrm{v} / \mathrm{v} \%$ methanol was similar to that of $75 \mathrm{v} / \mathrm{v} \%$ methanol, because their conductance was approximately equal (Figure 4B). The methanol LED sensor depended on the voltage input. Thus, we theoretically and experimentally observed the voltage (Table 1). We used 10 bit ADC that could recognize $0.005 \mathrm{~V}$ as a minimum voltage. 10 bit ADC converted experimental voltage presented in Table 1 into binary number in MCU. When the voltage input was larger than the binary number corresponding with $0 \mathrm{v} / \mathrm{v} \%$ methanol, LED sensor system recognized that solution did not contain methanol. It showed green LED color and FND system subsequently displayed number 0 . This LED sensor process was the same as 25 and $75 \mathrm{v} / \mathrm{v} \%$ methanol. Therefore, this methanol LED sensor could be a useful system to easily detect the methanol contents.

\section{Conclusion}

We developed the microfluidic LED sensor for methanol detection. The linear gradients of methanol were generated in the microfluidic device. The methanol harvested from the microfluidic device was analyzed by electrical conductivity and LED sensor. We demonstrated that the electrical conductivity was decreased with increasing the methanol and each percentage of the methanol was also observed by LED sensor. Therefore, this microfluidic LED device for methanol detection could be a powerful tool for methanol sensor applications.

\section{Additional file}

Additional file 1: C language program.

\section{Competing interests}

The authors declare that they have no competing interests.

\section{Authors' contributions}

THK and JML fabricated the LED sensor and microfluidic device to analyze methanol. BHC and BGC discussed the experimental data and wrote the paper. All authors read and approved the final manuscript.

\section{Acknowledgements}

This research was supported by BioNano Health-Guard Research Center funded by the Ministry of Science, ICT \& Future Planning (MSIP) of Korea as Global Frontier Project (Grant number H-GUARD_2014M3A6B2060503), Republic of Korea. This work was also supported by Leading Foreign Research Institute Recruitment Program through the National Research Foundation of Korea (NRF) funded by the Ministry of Science, ICT \& Future Planning (MSIP) (2013K1A4A3055268) and the Sogang University Research Grant of 2014 (Grant Number SRF-201414001).

Received: 30 December 2014 Accepted: 18 January 2015 Published online: 17 June 2015

\section{References}

1. H Guo, X Chen, Y Yao, G Du, H Li, Detection of ethanol and methanol vapors using polymer-coated piezoresistive Si bridge. Sensor Actuat. B-Chem. 155, 519-523 (2011)

2. Q Liu, JR Kirchhoff, Amperometric detection of methanol with a methanol dehydrogenase modified electrode sensor. J. Electroanal. Chem. 601, 125-131 (2007) 
3. LO Peres, RWC Li, EY Yamauchi, R Lippi, J Gruber, Conductive polymer gas sensor for quantitative detection of methanol in Brazilian sugar-cane spirit. Food Chem. 130, 1105-1107 (2012)

4. RX Wang, JJ Fan, YJ Fan, JP Zhong, L Wang, SG Sun, XC Shen, Platinum nanoparticles on porphyrin functionalized graphene nanosheets as a superior catalyst for methanol electrooxidation. Nanoscale 6, 14999-15007 (2014)

5. YL Hsin, KC Hwang, CT Yeh, Poly(vinylpyrrolidone)-modified graphite carbon nanofibers as promising supports for PtRu catalysts in direct methanol fuel cells. J. Am. Chem. Soc. 129, 9999-10010 (2007)

6. Z-B Wang, C-R Zhao, P-F Shi, Y-S Yang, Z-B Yu, W-K Wang, G-P Yin, Effect of a carbon support containing large mesopores on the performance of a Pt- Ru- Ni/C catalyst for direct methanol fuel cells. J. Phys. Chem. C 114, 672-677 (2009)

7. D-S Park, M-S Won, RN Goyal, Y-B Shim, The electrochemical sensor for methanol detection using silicon epoxy coated platinum nanoparticles. Sensor Actuat. B-Chem 174, 45-50 (2012)

8. J Li, L Dai, A hard modeling approach to determine methanol concentration in methanol gasoline by Raman spectroscopy. Sensor Actuator B. Chem. 173, 385-390 (2012)

9. Q Xu, Q Ye, H Cai, R Qu, Determination of methanol ratio in methanoldoped biogasoline by a fiber Raman sensing system. Sensor Actuator B. Chem. 146, 75-78 (2010)

10. D Jacobsen, KE McMartin, Antidotes for methanol and ethylene glycol poisoning. J. Toxicol. Clin. Toxicol. 35, 127-143 (1997)

11. J Brent, K McMartin, S Phillips, C Aaron, K Kulig, Fomepizole for the treatment of methanol poisoning. N. Engl. J. Med. 344, 424-429 (2001)

12. MB Gholivand, A Azadbakht, A nano-structured Ni(II)-chelidamic acid modified gold nanoparticle self-assembled electrode for electrocatalytic oxidation and determination of methanol. Mat. Sci. Eng. C. Mater. 32, 1955-1962 (2012)

13. JR Anderson, DT Chiu, H Wu, OJ Schueller, GM Whitesides, Fabrication of microfluidic systems in poly (dimethylsiloxane). Electrophoresis 21, 27-40 (2000)

14. Y Zhou, Q Lin, Microfluidic flow-free generation of chemical concentration gradients. Sensor Actuator B. Chem. 190, 334-341 (2014)

15. BG Chung, K-H Lee, A Khademhosseini, S-H Lee, Microfluidic fabrication of microengineered hydrogels and their application in tissue engineering. Lab Chip 12, 45-59 (2012)

16. Y-N Wang, R-J Yang, W-J Ju, M-C Wu, L-M Fu, Convenient quantification of methanol concentration detection utilizing an integrated microfluidic chip. Biomicrofluidics 6, 034111 (2012)

17. L-M Fu, W-J Ju, C-C Liu, R-J Yang, Y-N Wang, Integrated microfluidic array chip and LED photometer system for sulfur dioxide and methanol concentration detection. Chem. Eng. J. 243, 421-427 (2014)

18. QX Wu, TS Zhao, R Chen, WW Yang, A microfluidic-structured flow field for passive direct methanol fuel cells operating with highly concentrated fuels. J. Micromech. Microeng. 20, 045014 (2010)

19. Z Derlacki, AJ Easteal, AVJ Edge, LA Woolf, Z Roksandic, Diffusion coefficients of methanol and water and the mutual diffusion coefficient in methanol-water solutions at 278 and 298K. J. Phys. Chem. 89, 5318-5322 (1985)

20. BG Chung, F Lin, NL Jeon, A microfluidic multi-injector for gradient generation. Lab Chip 6, 764-768 (2006)

21. BG Chung, J Choo, Microfluidic gradient platforms for controlling cellular behavior. Electrophoresis 31, 3014-3027 (2010)

22. D Salvi, D Boldor, GM Aita, CM Sabliov, COMSOL Multiphysics model for continuous flow microwave heating of liquids. J. Food Eng. 104, 422-429 (2011)

23. K Higashikawa, Y Honda, M Inoue, T Kiss, N Chikumoto, N Sakai, T Izumi, H Okamoto, Investigation of three-dimensional current distribution at silver diffusion joint of RE-123 coated conductors based on magnetic microscopy combined with finite element method. IEEE Trans. Appl. Supercond. 21, 3403-3407 (2011)

24. S Ohshima, K Umezu, K Hattori, H Yamada, A Saito, T Takayama, A Kamitani, H Takano, T Suzuki, M Yokoo, S Ikuno, Detection of critical current distribution of YBCO-coated conductors using permanent magnet method. IEEE Trans. Appl. Supercond. 21, 3385-3388 (2011)
25. NG Patel, PD Patel, VS Vaishnav, Indium tin oxide (ITO) thin film gas sensor for detection of methanol at room temperature. Sensor Actuator B. Chem. 96, 180-189 (2003)

26. Y Long, T Wang, L Liu, G Liu, G Zhang, lon specificity at a low salt concentration in water-methanol mixtures exemplified by a growth of polyelectrolyte multilayer. Langmuir 29, 3645-3653 (2013)

\section{Submit your manuscript to a SpringerOpen ${ }^{\odot}$ journal and benefit from:}

- Convenient online submission

- Rigorous peer review

- Immediate publication on acceptance

- Open access: articles freely available online

- High visibility within the field

- Retaining the copyright to your article

Submit your next manuscript at $>$ springeropen.com 